\title{
Analysis and experiment of HBAR frequency spectra and applications to characterize the piezoelectric thin film and to HBAR design
}

\author{
*Shih-Yung Pao, Min-Chiang Chao, Zuoqing Wang, Chih-Hung Chiu, Kung-Chih Lan, Zi-Neng Huang, \\ Lung-Rung Shih, Chi-Lin Wang \\ TXC Corporation, Taoyuan County, Taiwan, R.o.C.
}

\begin{abstract}
In this paper, we present the resonance frequency spectra features of a composite resonator consisting of a piezoelectric film and a substrate plate. Based on those features, a direct method to characterize the piezoelectric film with two electrodes is presented. By directly using the parallel and series resonant frequency spectra of the HBAR, the electromechanical coupling factor, the density and the elastic constant of the piezoelectric film can be obtained directly. Some problems related to the accuracy of the method, especially the effect of the electrodes, are discussed.
\end{abstract}

\section{INTRODUCTION}

The most accurate measurement to characterize the acoustic properties of a piezoelectric film is the resonance frequency. A successful example is the IEEE standard on piezoelectricity [1], where the electromechanical coupling coefficient of a piezoelectric plate is determined solely by its fundamental resonant and anti-resonant frequencies. Piezoelectric films used at very high frequencies are too thin to be selfsupported, so they have to be deposited on a solid substrate and the film properties depend on the properties of the substrates. Thus, characterizations of the films have to be carried out on the composite resonators as shown in Fig. 1, and so a new measurement method is necessary. For the case where a piezoelectric ceramic film is deposited on an isotropic substrate to form a thickness extension mode resonator, some inversion methods [2], [3] had been developed to extract the electromechanical coupling coefficient of the film from the input impedance data. It is well known that the inversion procedures are not only time consuming but also not always reliable.

A direct method to characterize a piezoelectric film coated on an isotropic substrate to form a two-layer thickness expander composite resonator [4] was reported. By knowing the resonant spectrum of a composite resonator, three parameters of the piezoelectric film, i.e., the electro-mechanical coupling coefficient, the elastic constant, and the density could be determined. The validity of this method was demonstrated with simulations when electrodes were ignored. Recently, this method was developed to characterize the piezoelectric film operating in thickness shear mode by using the resonance frequency spectra of such a composite resonator [5]. Furthermore, the so called Resonance Spectra Method was extended to the case that the electrodes were taken into account [6] [7]. It can be identified, however, that the method is not complete up to now. Some factors affecting the accuracy have not been clarified, for example, the effects of the electrodes.

In this paper, the features of the resonance frequency of the HBAR will be analyzed firstly. Two sets of explicit formulae, which are the principles of this method, will be presented on assuming the materials loss-less and ignoring the mechanical effects of the electrodes. Validity of the method is identified by numerical simulation and some experimental results on $\mathrm{ZnO}$ /fused quartz HBAR. Explicit formulae to evaluate the electromechanical coupling coefficient, when the electrodes are taken into account, are obtained and comprehensive numerical simulations are carried out to show the effectiveness of the modified formula.

\section{The features OF THE RESONANCE FReQuency SPECTRA OF A HBAR}

Fig. 2 shows the input electric impedance of a two-layer HBAR as shown in Figl by ignoring the electrodes. This figure is calculated by using the following equation [4]

$$
Z_{\text {in }}=\frac{V}{I}=\frac{1}{j \omega C_{0}}\left[1-\frac{k_{t}^{2}}{\gamma} \cdot \frac{2 \tan (\gamma / 2)+z_{b} \tan \gamma_{b}}{1+z_{b} \tan \gamma_{b} / \tan \gamma}\right]
$$

where, $\gamma=2 \pi f \cdot l / c, \gamma_{b}=2 \pi f \cdot b / V_{b}$;

$C_{0}=\varepsilon_{33}^{s} \cdot A / l$ is the clamped capacitance of the resonator; $k_{t}^{2}=h_{33}^{2} \cdot \varepsilon_{33}^{s} / C_{33}^{D}$ is the thickness expander mode coupling factor of the piezoelectric film; $z_{b}=Z_{b} / Z_{0}, Z_{\mathrm{b}}$ and $Z_{0}$ are the acoustic impedance of the substrate plate and the film layer, respectively, and $Z_{b}=\rho_{b} \cdot V_{b} \cdot A, Z_{0}=\hat{\rho} \cdot \hat{V} \cdot A, \mathrm{~A}$ is the surface area of the resonator. $\rho_{b}, \hat{\rho}, V_{b}, \hat{V}$ are the densities and the extensional wave velocities in the $X_{3}$ direction of the substrate and the film, respectively; $l$ and $b$ are the thickness of the film and plate, respectively. The HBAR is consisting of a PZT film deposited on a stainless steel substrate. In the calculation, a small imaginary part is added to the velocities of both the film and substrate to avoid singularities.

It is shown in the input electric impedance of the HBAR that there are multiple sharp peaks and each corresponds a resonance "mode". More important features are the distributions of the resonance frequency spacing and they are analyzed as follows.

Based on the definition of the IEEE standard [1], the parallel resonant frequency of a single piezoelectric plate corresponds to the maximum resistance, which is the real part of the impedance of the resonator. By the same definition the parallel resonant frequencies can be calculated for all the modes. When the materials are loss-less, it is easy to find that the parallel resonant frequency equation is given by 


$$
\tan \gamma+z_{b} \tan \gamma_{b}=0
$$

The series resonant frequencies correspond to the maximums of the conductance, which is the real part of $Y_{\text {in }}=1 / Z_{\text {in }}$. They are the roots of the following equation for loss-less materials

$\tan \gamma+z_{b} \tan \gamma_{b}=\frac{k_{t}^{2}}{\gamma}\left[2 \tan (\gamma / 2)+z_{b} \tan \gamma_{b}\right] \tan \gamma$

After obtaining the resonance frequency data, by experiment or simulation, it is easy to calculate the spacing of the parallel resonant frequencies (SPRF) by defining

$$
\Delta f_{p}(m)=f_{p}(m+1)-f_{p}(m)
$$

Where, $f_{p}(m+1), f_{p}(m)$ are the parallel resonance frequencies of $(m+1)$-th and $m$-th orders of the HBAR.

By plotting $\Delta f_{p}(m)$ versus the frequency or the order $m$, a periodic distribution can be found and it is shown in Fig. 3 . There are four unique values in the figure: (1) the periodicity, $\Delta f_{C}$; (2) the SPRF value at the center of the regular regions, $\Delta f_{N}$; (3) the SPRF value at the center of the transition regions, $\Delta f_{T}$; (4) the modal frequency spacing of the bare plate, $\Delta f_{0}$. Later, we will derive the formulae which relate these unique SPRF values and the associated parameters $\hat{\rho}$ and $\hat{V}$ or $\hat{C}_{33}^{D}$. In other hand, from the definition of effective coupling coefficient $k_{e f f}^{2}(m)$

$$
k_{e f f}^{2}(m)=\frac{\pi^{2}}{4} \cdot \frac{f_{s}(m)}{f_{p}(m)} \cdot\left[1-\frac{f_{s}(m)}{f_{p}(m)}\right]
$$

where $f_{p}(m), f_{s}(m)$ are the $m$-order parallel and series resonant frequencies, we can obtain the effective electro-mechanical coupling factors of each mode. Plotting $k_{e f f}^{2}(m)$ versus the order $m$ or the frequency, we can obtain a distribution as shown in Fig. 4. Simulation results showed that the acoustic impedance ratio of the two layers significantly affects the distribution of the $k_{e f f}^{2}$ versus the mode order (or frequency). For the porous PZT on stainless steel plate sample, where $z_{b}=Z_{b} / Z_{0} \gg 1$, as shown in Fig.4, there is a peak value of the $k_{e f f}^{2}$ located at the center of the transition region, which corresponds to a frequency $f=\hat{V} / 4 l$. It seems that the piezofilm is working as a "quarter wavelength resonator". Contrarily, for the case of $\mathrm{ZnO}$ film on a fused quartz plate resonator, where $z_{b}<<1$, the peak $k_{\text {eff }}^{2}$ value is located at the frequency $f=\hat{V} / 2 l$, and the film acts as a "half wavelength resonator". In next section, we will derive the explicit formulae, which are the principles of the method, to determine the $k_{t}^{2}$ of the piezoelectric film based on the $k_{e f f}^{2}(m)$ distribution.

Briefly, the SPRF, i.e., $\Delta f_{p}(m)$ and the $k_{e f f}^{2}(m)$ of a composite resonator are determined by the acoustic properties and geometric parameters of the piezoelectric film and the substrate of the HBAR. Both have their maximum magnitude at a certain frequency, which is determined mainly by the piezoelectric film and also by the acoustic impedance ratio of the piezoelectric film and the substrate. When the acoustic impedance of the piezoelectric layer is smaller than that of the substrate, referred to as a "hard substrate", such as PZT on stainless steel, the maximum magnitude is located in the first transition region. When the acoustic impedance of the piezoelectric layer is greater than that of the substrate, referred to as a "soft substrate", such as $\mathrm{ZnO}$ on quartz, the maximum magnitude is located in the first normal region. In the following sections, we will present the relations between the resonant frequencies of the modes and the piezoelectric film parameters on the approximations of ignoring the effects of the electrodes and material loss. Since the film parameters can be determined by the distribution of the resonant frequencies merely, we name this method as Resonant Spectrum Method.

\section{PRINCIPLES OF THE RESONANT SPECTRUM METHOD}

The principles of the Resonant Spectrum Method are based on two groups of explicit approximate formulae- (a) The relationship of three SPRF unique values and the density, $\hat{\rho}$, and the elastic constant, $\hat{C}_{33}^{D}$, of the piezoelectric film; (b) The relationship of the effective coupling factor, $k_{e f f}^{2}(m)$ of a specific mode, and $k_{t}^{2}$ of the piezoelectric film.

\section{(1) Principles of the Resonance Spectrum Method to determine $\hat{\rho}$ and $\hat{C}_{33}^{D}$}

It was found that the distribution of the SPRF is the same as the modified modal frequency spacing (MMFS) method $[9,10]$, and has definite relations with the two parameters of the piezoelectric film $[6,7]$. The results can be summarized as follows.

(a). At the center of the regular regions, where $\gamma \approx n \pi$, $(n=0,1,2, \ldots)$, the SPRF value can be derived from (2) and is given approximately by

$$
\Delta f_{N} \approx \Delta f_{0} \cdot\left(1+\frac{2 \cdot(\hat{\rho} l)}{\rho_{b} b}\right)^{\frac{-1}{2}} .
$$

Where:

$$
\Delta f_{0}=V_{b} / 2 b
$$

is the mechanical resonance frequency of the fundamental mode, or the spacing of its modes of the bare plate. It is assumed that all the parameters of the substrate are known, and so Eq. (6) indicated that $\Delta f_{N}$ only depends on $\hat{\rho} l$. By evaluating the value of $\Delta f_{N}$ from the SPRF data, the value $\hat{\rho} l$ can be determined. 
(b). At the center of the transition regions, where $y \approx\left(n+\frac{1}{2}\right) \pi$, the SPRF value can be derived from (2) and given by

$$
\Delta f_{T} \approx \Delta f_{0} \cdot\left(1+\frac{\rho_{b} V_{b}^{2}}{b} \cdot \frac{l}{\hat{C}_{33}}\right)^{-1}
$$

It is indicated that $\Delta f_{T}$ only depends on $\left(l / \hat{C}_{33}\right)$. By evaluating $\Delta f_{T}$ from the SPRF data, the value $\left(l / \hat{C}_{33}\right)$ can be determined.

(c). The period of the SPRF distribution is obtained from (2) and given by

$$
\Delta f_{C}=\frac{\hat{V}}{2 l}=\frac{1}{2} \cdot \sqrt{\frac{\left(\hat{C}_{33} / l\right)}{(\hat{\rho} l)}} .
$$

The value of the period of SPRF depends only on $\hat{V}$. By evaluating $\Delta f_{C}, \hat{V}$ can be obtained directly. Either $\left(l / \hat{C}_{33}\right)$ or $\hat{\rho} l$ is known, the other one can be calculated from this formula.

The three formulae, (6), (8) and (9), give an approximate relation between the material and geometrical parameters of the two layers and the three unique values, $\Delta f_{N}$, $\Delta f_{T}$ and $\Delta f_{C}$. The three unique values can be evaluated from the SPRF distribution, which are acquired from experimental data. The parameters involved in the three formulae are the densities, $\rho$ and $\rho$, bulk modulus, $\left(\lambda_{1}+2 \mu_{1}=\rho_{b} V_{b}^{2}\right)$ and $C_{33}$, (or longitudinal wave velocities, $V_{b}$ and $\hat{V}$ ), and thickness, $b$ and $l$, of the two layers, respectively. In some cases, three unknown parameters can be evaluated from those formulae. But for the case discussed here (the three parameters of the substrate plate are known and the three parameters of the ceramic film are unknown), only two of the three unknown parameters can be evaluated from those formulae, because they are always in the combined forms of ( $l / \hat{C}_{33}$ ) and $\hat{\rho} l$. So in the following simulations, the thickness of the film is given and only two parameters $C_{33}$ and $\rho$ will be determined.

(2) Principles of the Resonance Spectrum Method to determine $k_{t}^{2}$

Using the definition of the "effective coupling factor" given in Eq.(4) and the resonance frequency equations (2) and (3), we derived two explicit formulae which relate $k_{t}^{2}$ to the "effective coupling factor" for a special mode at the center of the first normal region, $k_{e f f}^{2}\left(m_{N}\right)$, and at the first transition region, $k_{e f f}^{2}\left(m_{T}\right)$, respectively.

At the first normal region where $\tan (\gamma) \approx 0$, we can write $\gamma=\pi+\varepsilon$ and $\gamma_{b}=m_{N} \pi+\delta$, and ignore the term containing $\varepsilon \cdot \delta$ to obtain the formula

$$
k_{t}^{2}=\left(1+\rho_{b} b / \hat{\rho} l\right) \cdot\left(k_{e f f}^{2}\left(m_{N}\right)\right)
$$

where $m_{N}=\operatorname{round}\left(\hat{V b} / V_{b} l\right)+1$, and it is the mode order at the center of the region.

At the first transition region where $\tan (\gamma) \rightarrow \infty$, we can express $\gamma=(1 / 2) \cdot \pi+\varepsilon ; \quad$ and $\gamma_{b}=\left(m_{T}+1 / 2\right) \pi+\delta$. After some algebraic manipulation, we obtain the formula

$k_{t}^{2}=\left[1+(b / l) \cdot\left(C_{33}^{D} / \rho_{b} \cdot V_{b}^{2}\right)\right] \cdot \frac{1}{\Gamma} \cdot\left(k_{e f f}^{2}\left(m_{T}\right)\right)$

where $m_{T}=\operatorname{round}\left[\hat{V} b /\left(2 V_{b} l\right)+1 / 2\right]$ and it is the mode order at the center of the region.

$\Gamma=1+2 \cdot \frac{\hat{\rho} \hat{V}}{\rho_{b} V_{b}} \cdot(1+\varepsilon) \cdot \delta$

is a correction factor which is not necessarily near unity when $m_{T}$ is not very large (50, for example).

Formulae (10) and (11) express the principles of the measurement method--the $k_{t}^{2}$ value of the film can be determined from the $k_{e f f}^{2}\left(m_{N}\right)$ and the mass ratio of the two layers, or from the $k_{e f f}^{2}\left(m_{T}\right)$, and the thickness and elasticity of the two layers. It is interesting to note that in (10) the relation between $k_{t}^{2}$ and $k_{e f f}^{2}$ is engaged by the distribution of the vibration kinetic energy and in (11) the relation is engaged by the elastic potential energy distribution. The effective coupling factors $k_{e f f}^{2}\left(m_{N}\right)$ and $k_{e f f}^{2}\left(m_{T}\right)$ are measured by using the resonance frequency spectra while the mass ratio, elastic and geometry parameters of the two layers are previously known.

It should be kept in mind that the two groups of explicit formulae were derived under two assumptions -- (a) The materials are loss-less and so the parallel and series resonance frequency equations are given by (2) and (3) and (b) the effects of the electrodes are ignored. Actually, there are some losses in materials always, and the resonance frequencies are determined by Eq.(1) directly. The validity of the method has to be identified. In the following two sections, we will present numerical simulation results and some experimental results to show the validity of the method and the effects of the electrode on the accuracy of the method. 


\section{VALIDITY OF THE METHOD}

It is understood that the two formulae were derived by using Eqs.(2) and (3) which pertain only to loss-less materials. Any piezoelectric material, however, has mechanical, dielectric and piezoelectric losses. As such, the resonance frequencies have to be calculated directly from Eq.(1) rather than from (2) and (3). For checking the validity of the method to determine the $k_{t}^{2}$, we carried out a numerical simulation by taking the mechanical losses into account to show its effects on the $k_{t}^{2}$ accuracy. Another part of the method, i.e., determining the density and elastic constant by the SPRF distribution, had been analyzed elsewhere [10].

(1). On samples of a kind of special PZT film on stainless steel subst Taking the velocity in PZT film by adding an imaginary part to represent its loss, i.e., $\hat{V}=c_{r}+j \cdot c_{i}$, we carried out the simulations. We took $\mathcal{c}_{r}=2.4 \mathrm{~km} / \mathrm{s}$ and $c_{i} / c_{r}=0.0,0.1 \%, 1.0 \%, 2.0 \%, 5.0 \%$, and $10.0 \%$ in our calculations. Based on the IEEE Standard the parallel resonance frequencies are calculated when the real part of the impedance goes to a maximum and the series resonance frequencies are calculated when the real part of the admittance goes to a maximum. These definitions are independent of the lumped-parameter equivalent circuit. By using Eq.(1), the first few tens of pairs of resonant frequencies are calculated, then from (5), (10) and (11), the electromechanical coupling coefficient, $k_{t}^{2}$, of the film was obtained. It was found that if the imaginary part of the elastic constant is less than $5.0 \%$, which corresponds to the phase angle of the elastic constant of 3.0 degrees, the resonant frequencies and so the $k_{t}^{2}$ values do not change significantly (less than $1.0 \%$ ). This means that the proposed method is available for real piezoelectric films if the phase angle of the elastic constant is not more than 3 degrees, which is a reasonable criterion for the common piezoelectric films. The sample used in the simulation is a thick film, since the relative thickness in wavelength of the film to the substrate is about $1 / 20$.

\section{(2). On samples of $\mathrm{ZnO} / \mathrm{SiO}_{2} \mathrm{HBAR}$}

It was shown [7] that when the mechanical loss changes up to 20 times, the results of the SPRF and the effective electromechanical coupling factors have no significant changes, and the errors of the simulated results are within $2 \%$. It means the method is validid for practical materials.

Experimental results of a few $\mathrm{ZnO} /$ fused quartz [7] showed the results are reasonable. Fig. 5 and 6 are the SPRF distribution and $k_{e f f}^{2}(m)$ distribution versus frequency. The thickness of the $\mathrm{ZnO}$ film is around $4.8 \mu \mathrm{m}$ and of the quartz substrate $62 \mathrm{mil}$. There is a circular ground electrode of aluminum approximately $400 \mathrm{~nm}$ thick underlying the $\mathrm{ZnO}$ film, and there are 4 small circular electrodes of $400 \mathrm{~nm}$ aluminum on the top of $\mathrm{ZnO}$ film, forming $4 \mathrm{HBAR}$ devices. It was shown in Fig. 5 that the distribution of SPRF is regular but the data are dispersive and so a kind of data fitting is necessary. It is interesting to note Fig.6 that the distribution of $k_{e f f}^{2}(m)$ is regular and very smooth and so data fitting is un-necessary. The jump in Fig.6, which always appears for all the HBAR devices, is caused by measurement system. Because only the maximum value and corresponding position are concerned in the method, the jump as shown in Fig. 6 will not affect the result. The experimental results of the three parameters obtained by this method are: $\hat{\rho}=5.29 \mathrm{~g} / \mathrm{cm}^{3}$, $\hat{C}_{33}^{D}=19.9\left(10^{10} \mathrm{~N} / \mathrm{m}^{2}\right), \quad k_{t}^{2}=7.6 \%$. These values are reasonable by comparing with the data of bulk $\mathrm{ZnO}$ crystal $\left(\hat{\rho}=5.675 \mathrm{~g} / \mathrm{cm}^{3}, \quad \hat{C}_{33}^{D}=20.4\left(10^{10} \mathrm{~N} / \mathrm{m}^{2}\right), \quad\right.$ and $k_{\mathrm{f}}^{2}=8.0 \%$.) and it indicated that the method is practical.

However, it has to be kept in mind that the position on abscissa, where the maximum $k_{e f f}^{2}(m)$ is, is not necessarily being
strate. the order $m_{N}$ or $m_{T}$ as given in Eq.(10) and (11). There is no analytical identification and it is necessary to identify their coincidence (or not) by numerical simulation.

\section{(3). Deviation of the maximum value position of $k_{e f f}^{2}(m)$}

\section{from order $m_{N}$}

It is the most easy way to find the position and value of the maximum $k_{e f}^{2}(m)$ both in experiment and simulation. But in formula the value $k_{e f f}^{2}\left(m_{N}\right)$ is at the center of regular region, i.e., for the order of $m_{N}$, as given in (10), for the case of $\mathrm{ZnO}$ film on fused quartz substrate. Contrarily, for the case of PZT on steel, the maximum value $k_{e f f}^{2}(m)$ is at the center of the transition region, i.e., at the order of $m_{T}$. But this relation can't be analyzed and hasn't numerically confirmed up to now. In this section we identify the relation of them by numerical simulations.

Two kinds of piezoelectric films and three kinds of substrates are chosen to cover different acoustic impedance ratios, $\rho_{b} V_{b} / \hat{\rho} \hat{V}_{L}$, as shown in Table I. For the cases $\rho_{b} V_{b} / \hat{\rho} \hat{V}_{L}>1$, it is the "hard substrate" case, while the case $\rho_{b} V_{b} / \hat{\rho} \hat{V}_{L}<1$ is the "soft substrate". The data of the material parameters are listed in Table II. Thickness of the piezoelectric films are fixed-for $\mathrm{ZnO}$ Film, $l=4.808 \mu \mathrm{m}$ and for PZT film, $l=2.50 \mu \mathrm{m}$. The thickness of the substrates is chosen to $N=\Delta f_{C} / \Delta f_{0}=\left(\hat{V} / V_{b}\right) \cdot(b / l)$ being an integer. For fused quartz, $b=1.5125 \mathrm{~mm}(\mathrm{~N}=312$ and 240), for silicon, $b=1.4912 \mathrm{~mm}(\mathrm{~N}=208$ and 160$)$ and for steel, $b=1.1377$ $(\mathrm{N}=260$ and 200).

For a given combination, one can use Eq.(1) to calculate the resonance frequencies of the first $2 \mathrm{~N}$ modes, and then use Eq. (5) to calculate $k_{e f f}^{2}(m)$ for all the modes. Plotting $k_{e f f}^{2}(m)$ versus mode order, one can identify if the maximum of $k_{e f f}^{2}(m)$ is coincident with the order $m_{N}$ for the "soft substrate" cases, and with the order $m_{T}$ for the "hard substrate" cases.

The simulation results for the six different combinations are shown in table 4 . It is clearly indicated that when $\rho_{b} V_{b} / \hat{\rho} \hat{V} \ll 1$, i.e., for very soft substrates, the maximum 
$k_{\text {eff }}^{2}(m)$ position is coincident with $m_{N}$. The smaller the $\rho_{b} V_{b} / \hat{\rho} \hat{V}$ value, the closer the position of the maximum $k_{e f f}^{2}(m)$ to $m_{N}$ is. Contrarily, when $\rho_{b} V_{b} / \hat{\rho} \hat{V} \gg>1$, i.e., for very hard substrates, the maximum $k_{\text {eff }}^{2}(m)$ position is coincident with $m_{T}$. The larger the $\rho_{b} V_{b} / \hat{\rho} \hat{V}$ value, the closer the position of the maximum $k_{e f f}^{2}(m)$ to $m_{T}$ is. For the case $\rho_{b} V_{b} / \hat{\rho} \hat{V}=1$, the maximum $k_{e f f}^{2}(m)$ is at the mid between $m_{N}$ and $m_{T}$.

The simulation results give us a few pieces of important information. For the samples of soft PZT film on steel [4], the maximum $k_{e f f}^{2}(m)$ position is very close to $m_{T}$ ( $2 \%$ error), and it won't cause visible error. For the samples of $\mathrm{ZnO}$ on fused quartz, the maximum $k_{\text {eff }}^{2}(m)$ position is close to $m_{N}(5 \%$ deviation). If we check Fig. 6 and the related data carefully, the 5 $\%$ deviation will cause the mode-order difference of about 10 and which corresponds to around $6 \%$ decrease of $k_{e f f}^{2}(m)$ value. This means the evaluated value of $k_{t}^{2}=7.6 \%$ should be modified to $7.2 \%$.

\section{EFFECTS OF THE ELECTRODE}

Obviously, for the high frequency case the piezoelectric film is very thin and the effects of the electrodes can't be ignored. The effects were discussed primarily by Zhang et al $[7,8]$. In this section, we present a comprehensive simulation for the electrode effects on the accuracy of the method and the modified formula. given by

The input electrical impedance of a four layer HBAR is

$Z_{\text {in }}=\frac{V}{I}=\frac{1}{j \omega C_{0}} \cdot\left[1-\frac{k_{t}^{2}}{\gamma} \cdot \frac{\left(z_{1}+z_{2}\right) \cdot \sin \gamma+j \cdot 2(1-\cos \gamma)}{\left(z_{1}+z_{2}\right) \cdot \cos \gamma+j \cdot\left(1+z_{1} z_{2}\right) \cdot \sin \gamma}\right]$

where

$$
\begin{aligned}
& z_{1}=Z_{1} / Z_{0} \text { and } z_{2}=Z_{2} / Z_{0}, \text { and } \\
& Z_{1}=j Z_{e 1} \tan \gamma_{e 1} \\
& Z_{2}=j \frac{Z_{s b} \tan \gamma_{s b}+Z_{e 2} \tan \gamma_{e 2}}{1-\left(Z_{s b} / Z_{e 2}\right) \tan \gamma_{e 2} \tan \gamma_{s b}} \\
& \gamma_{e 1}=\frac{\omega d_{1}}{V_{e 1}} ; \gamma_{e 2}=\frac{\omega d_{2}}{V_{e 2}} ; \gamma_{s b}=\frac{\omega b}{V_{s b}} ; \\
& Z_{e 1}=A \cdot \rho_{e 1} \cdot V_{e 1} ; Z_{e 2}=A \cdot \rho_{e 2} \cdot V_{e 2} \\
& Z_{s b}=A \cdot \rho_{s b} \cdot V_{s b}
\end{aligned}
$$

Using similar procedure as the two-layer case, we obtained a formula based on the formula developed by Zhang [8]. In Zhang's model, the effect of the mid electrode is divided into two parts: half into film, and another half into the substrate. We consider the complete mid-layer engaged into the thin film and so the formula is modified to be

$$
k_{t}^{2}=\frac{\left(1+\rho_{s b} l_{s b} / \rho l\right)}{\left(1+\rho_{e l} l_{e l} / \rho l+\rho_{e 2} l_{e 2} / \rho l\right)^{2}} \cdot k_{e f f}^{2}\left(m_{N}\right)
$$

We carried out a comprehensive simulation to verify the validity of the model. The data of the parameters used in simulation are listed in Table 2. The samples cover a wide range of $\rho_{b} V_{b} / \hat{\rho} \hat{V}_{L}$ as shown in Table 1. Eq.(18) clearly showed that the effects of the electrodes are mass loading only, but this conclusion has to be verified. So we choose gold and tungsten as the electrode because they have similar density but very different acoustic impedance. The maximum mass ratio of the electrode to the film covers from $7.9 \%$ to $85.5 \%$, as shown in Table 3 , on the condition of keeping the thickness of the electrode less than $5 \%$ of wavelength (to guarantee $\tan (\alpha) \approx \alpha$, i.e., merely a mass loading).

Taking a set of data of a specified combination, the resonance frequencies of the modes within the first period are calculated by using the impedance equation (13). From those resonant frequencies of the modes, one can obtain the $k_{\text {eff }}^{2}(m)$ by (5), and further the values of the electro-mechanical coupling coefficient, $k_{t}^{2}$, of the film can be evaluated by Eq. (18) and Eq.(10). Comparing the evaluated values of $k_{t}^{2}$ with the input data, the errors can be evaluated. The trend of the errors versus the thickness of electrode is indicated by two curves obtained by Eq. (10) and (18), respectively. In Fig.7, the effects of three kinds of electrodes for a combination of a film on a substrate are shown by a figure. The bold lines are the results from the non-corrected formula (10) and the fine lines are the results from the modified formula (18). It is clearly shown that the effectiveness of the modified formula (18) is satisfactory. The formula is still valid even for the mass loading as large as $85 \%$ of the film's mass. Besides, it is identified that the effect of the electrode is merely a kind of mass loading. The effects are the same for gold and tungsten at the same mass ratio. There is a little over correction for the $\mathrm{ZnO}$ film on the three substrates but there is no this phenomenon for PZT film. There is an error of no more than $3 \%$ even at the case of ignoring the electrode's effect. Those inaccuracies, however, are allowable for determining the electromechanical coupling coefficients.

\section{Conclusions AND discussions}

The principles of the Resonant Spectrum Method were presented briefly. In this method three major parameters of piezoelectric films, i.e., the electromechanical coupling coefficient, the elastic constant and density, can be obtained by using a set of explicit formulae. Numerical simulation and experimental results on $\mathrm{ZnO}$ /fused quartz HBAR confirmed the validity.

Two problems relating with the validity and accuracy of the method are investigated in detail. The first problem is the relation of the mode order, where the effective coupling is in maximum, and the specific order $m_{N}$ and $m_{T}$. Simulation results for the six different combinations showed that when $\rho_{b} V_{b} / \hat{\rho} \hat{V} \ll<$, i.e., for very soft substrates, the maximum 
$k_{\text {eff }}^{2}(m)$ position is coincident with $m_{N}$. The smaller the $\rho_{b} V_{b} / \hat{\rho} \hat{V}$ value, the closer the position of the maximum $k_{e f f}^{2}(m)$ to $m_{N}$ is. Contrarily, when $\rho_{b} V_{b} / \hat{\rho} \hat{V} \gg 1$, i.e., for very hard substrates, the maximum $k_{e f f}^{2}(m)$ position is coincident with $m_{T}$. The larger the $\rho_{b} V_{b} / \hat{\rho} \hat{V}$ value, the closer the position of the maximum $k_{e f f}^{2}(m)$ to $m_{T}$ is. For the case $\rho_{b} V_{b} / \hat{\rho} \hat{V}=1$, the maximum $k_{e f f}^{2}(m)$ is at the mid between $m_{N}$ and $m_{T}$.

The second problem is the effects of the electrodes and how to correct the effects. We introduced a modified formula based on [8], and the simulation results indicated that the modified formula gave satisfactory correction.

An obvious advantage of the Resonant Spectrum Method is its "directness". Usually, the parameters of the piezoelectric films are determined from their electric features measurements, for example, from the input impedance by an inversion method. Besides, it is very important in the developed method that resonant frequency is the only measurant and the distributions of resonant frequencies are of interest merely. Therefore, the accuracy of the measurant is guaranteed and the calibration of the measurement system is not critical, while the accuracy of the calibration makes a great difference in the measurement of the impedance. This is not trivial because usually accurate calibration is very difficult.

There are some limits on this method. The thickness of the film has to be known and actually, the error in determining the thickness of the film is the major source to evaluate the three parameters of the piezoelectric films.

The formulae, (6), (8), (10) and (11), are useful for designing HBAR. Those formulae give the spacing of the modes, the effective coupling factor, and the related parameters explicitly.

\section{ACKNOWLEDGMENT}

The authors would highly appreciate Dr. Y. Zhang for his generosities to allow us quoting his experimental results.

\section{REFERENCES}

[1] IEEE Standard on Piezoelectricity, ANSI/IEEE Std 176-1987.

[2] F.S. Hickernell, "Measurement techniques for evaluating piezoelectric thin films", Proc. 1996 IEEE Ultrasonics Symposium, 235-242, (San Antonio, 1996).

[3] B.S. Naik, J. J. Lutsky, R. Rief and C. D. Sodini, "Electromechanical coupling constant extraction of thin-film piezoelectric materials using a bulk acoustic wave resonator", IEEE Trans. on UFFC, Vol. 45(1), 257-263, 1998.

[4] Z. Wang, Y. Zhang, and J.D.N. Cheeke, "Characterization of electromechanical coupling coefficient of piezoelectric film using composite resonators", IEEE Trans. UFFC, Vol 46 (5), pp. $1327-$ 1330,1999

[5]. M.-C. Zhao, B. Wu, Z. Wang and C.-L, Wang, "Characterizing the thickness shear mode properties of the piezoelectric thin films deposited on substrates", 200I IEEE Ultrasonic Symposium, (Atlanta, Georgia, US), P2P-1, 2001.

[6] Y.Zhang, Z. Wang, J.D.N Cheeke and F.S. Hickernell, "Direct characterization of $\mathrm{ZnO}$ films in composite resonators with the resonance spectrum method", Proc. 1999 IEEE Ultrasonic Symposium, (Lake Tahoe, Nevada, US, 1999), pp. 991-994, 1999.

[7] Y. Zhang, Z. Wang, and J.D.N. Cheeke, "Simulation of electromechanical coupling coefficient by modified modal frequency spectrum method including the electrode effect", Ultrasonics, Vol.38, pp. 114-117, 2000.

[8]. Y. Zhang, "Resonant spectrum method to characterize piezoelectric films in composite resonators", Thesis In The Department of Physics, For the degree of Doctor of Philosophy at Concordia University, Montreal, Quebec, Canada, February 2002, (C) Yuxing Zhang, 2002.

[9] Z. Wang, X. Li and D. N. Ckeeke, "A modified modal frequency spacing method for coating characterization", $J$. Acoust. Soc. Am., Vol. 104 (5), pp. 3199-3122, 1998.

[10] Z. Wang and J. D. N. Cheeke, "Characterizing unpoled piezoelectric ceramic films by Lamb waves", IEEE Trans. UFFC, Vol. 46 (5), pp. 1094-1100, 1999.

*e-mail:sypao@txc.com.tw

+e-mail: chaomk@txc.com.tw

Table 1 : Impedance ratio $\rho_{b} V_{b} / \hat{\rho} \hat{V}_{L}$ :

\begin{tabular}{|c|c|c|c|}
\hline & $\begin{array}{c}\text { Fused } \\
\text { Quartz }\end{array}$ & Silicon & Steel \\
\hline ZnO & 0.47 & 0.61 & 1.25 \\
\hline PZT-film & 0.95 & 1.24 & 2.54 \\
\hline
\end{tabular}

Table 2 : Material parameters:

\begin{tabular}{|c|c|c|c|c|c|c|c|}
\hline & Density & $\mathrm{C}_{33}$ & $\mathrm{C}_{44}$ & $\mathrm{~V}_{\mathrm{L}}$ & $\mathrm{V}_{\mathrm{s}}$ & $\rho \mathrm{V}_{\mathrm{L}}$ & $\rho \mathrm{V}_{\mathrm{S}}$ \\
\hline $\mathrm{ZnO}$ & 5.675 & 204.3 & 46.5 & 6.00 & 2,862 & 34.05 & 16.24 \\
\hline $\begin{array}{c}\text { PZT- } \\
\text { film** }\end{array}$ & 7.0 & 40.32 & 22.68 & 2,400 & 1,800 & 16.80 & 12.60 \\
\hline $\begin{array}{c}\text { Fused } \\
\text { Quartz }\end{array}$ & 2.65 & 97.0 & 44.4 & 6,050 & 4,093 & 16.033 & 10.846 \\
\hline Silicon & 2.33 & 186.5 & 66.5 & 8,947 & 5,342 & 20.846 & 12.447 \\
\hline Steel & 7.80 & 232.6 & 124.8 & 5.231 & 4.00 & 42.596 & 31.200 \\
\hline $\mathrm{Al}$ & 2.70 & 111.3 & 26.1 & 6,422 & 3,109 & 17.339 & 8.394 \\
\hline $\mathrm{Au}$ & 19.49 & 220.2 & 29.9 & 3,361 & 1,239 & 65.506 & 24.148 \\
\hline $\mathrm{W}$ & 18.71 & 512.0 & 153.0 & 5,231 & 2,860 & 97.820 & 53.511 \\
\hline
\end{tabular}


Piezoelectric film:

ZnO Film: $l=4.808 \mu \mathrm{m}$;

$k_{t}^{2}=0.080 ; \quad k_{15}^{2}=0.029$;

High-temperature PZT--thin film: $l=2.50$ $\mu \mathrm{m} ; k_{t}^{2}=0.04 ; \quad k_{15}^{2}=0.02$;

\section{Substrate:}

Fused quartz: $b=1.5125 \mathrm{~mm}$;

Silicon: $b=1.4912 \mathrm{~mm}$;

Steel: $b=1.1377 \mathrm{~mm}$;

\section{Electrode thickness:}

$l_{e}=0.01,0.02,0.05,0.10,0.20,0.30$ and 0.40 $\mu \mathrm{m}$;

Table 3: The Maximum values of $2 \cdot \rho_{E} d_{E} / \hat{\rho} l$ :

\begin{tabular}{|l|l|l|l|l|l|}
\hline Al/Zno & Au/ZnO & W/ZnO & Al/PZT & Au/PZT & W/PZT \\
\hline $7.9 \%$ & $28.6 \%$ & $54.8 \%$ & $12.3 \%$ & $44.6 \%$ & $85.5 \%$ \\
\hline
\end{tabular}

Table 4:

\begin{tabular}{|c|c|c|c|}
\hline & $\rho_{b} V_{b} / \hat{\rho} \hat{V}_{L}$ & $m \rightarrow\left(k_{\text {eff }}^{2}\right)_{\text {Max }}$ & $m \rightarrow\left(k_{\text {eff }}^{2}\right)_{\text {Max }}$ \\
\hline $\begin{array}{c}\text { ZnO/ } \\
\text { Fused quartz }\end{array}$ & 0.47 & $1.90 m_{T}$ & $0.95 m_{N}$ \\
\hline ZnO/Si & 0.61 & $1.83 m_{T}$ & $0.92 m_{N}$ \\
\hline $\begin{array}{c}\text { PZT/ } \\
\text { Fused quartz }\end{array}$ & 0.95 & $1.52 m_{T}$ & $0.76 m_{N}$ \\
\hline $\begin{array}{c}\text { PZT/Si } \\
\text { ZnO/steel }\end{array}$ & 1.24 & $1.21 m_{T}$ & $0.61 m_{N}$ \\
\hline PZT/steel & 2.54 & $1.02 m_{T}$ & $0.51 m_{N}$ \\
\hline
\end{tabular}

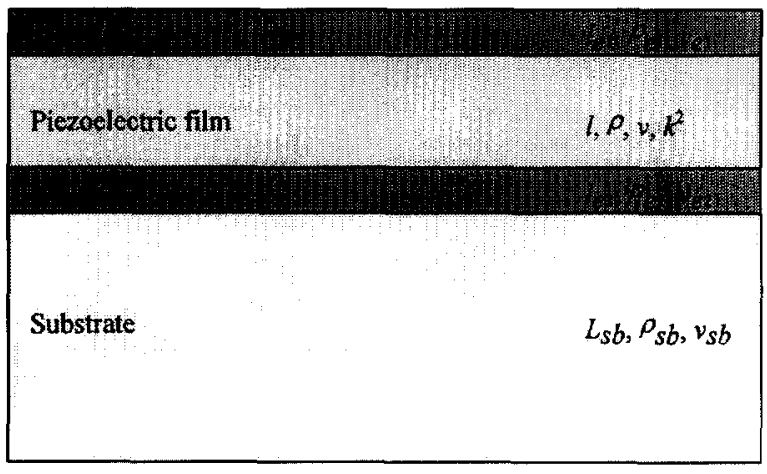

Fig 1. Configuration of a HBAR.

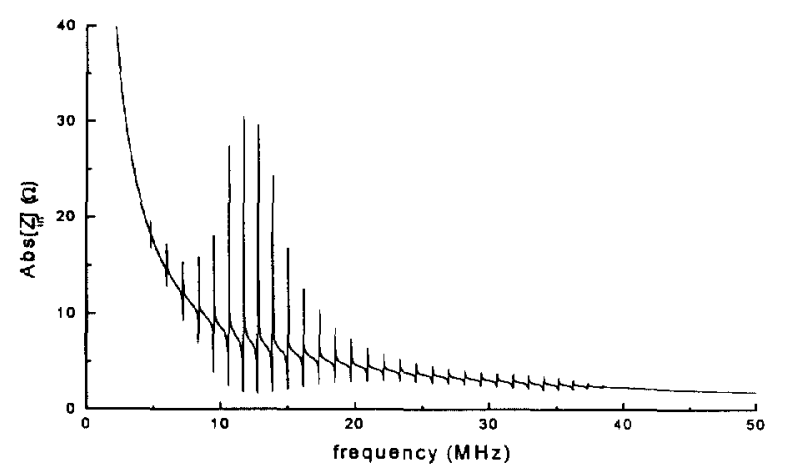

Fig 2. The impedance magnitude of a HBAR.

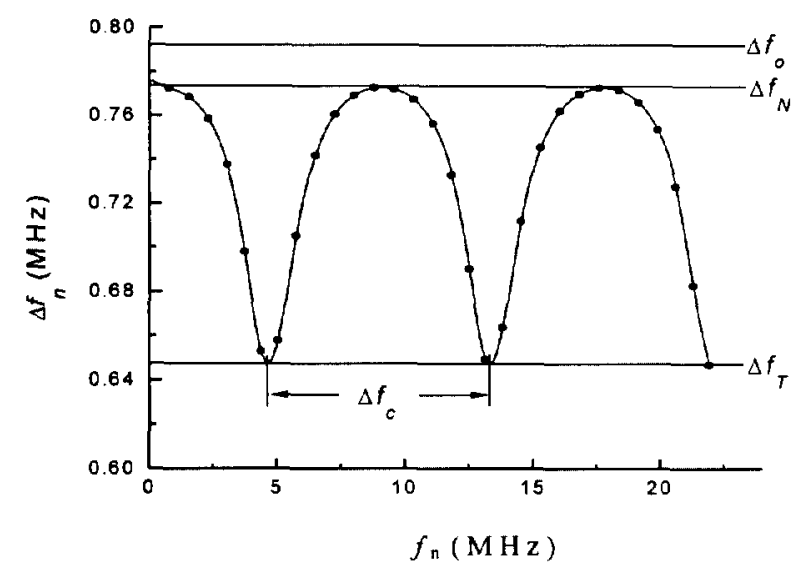

Fig 3. SPRF distribution versus mode order. 


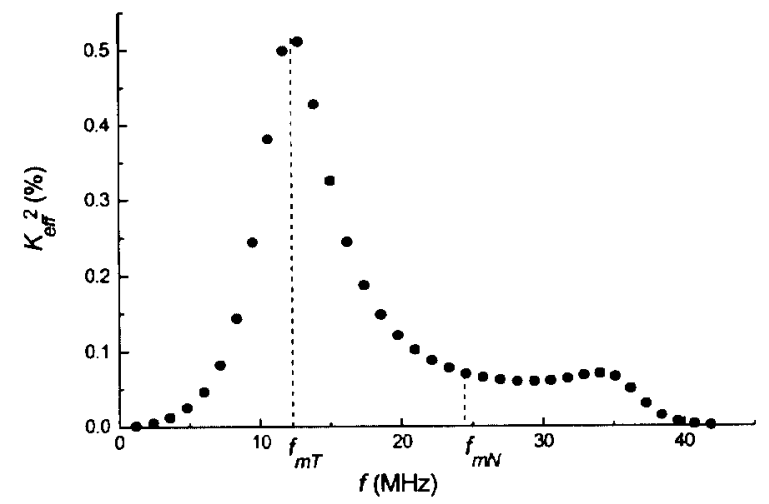

Fig 4. $k_{e f f}^{2}(m)$ distribution versus mode order.

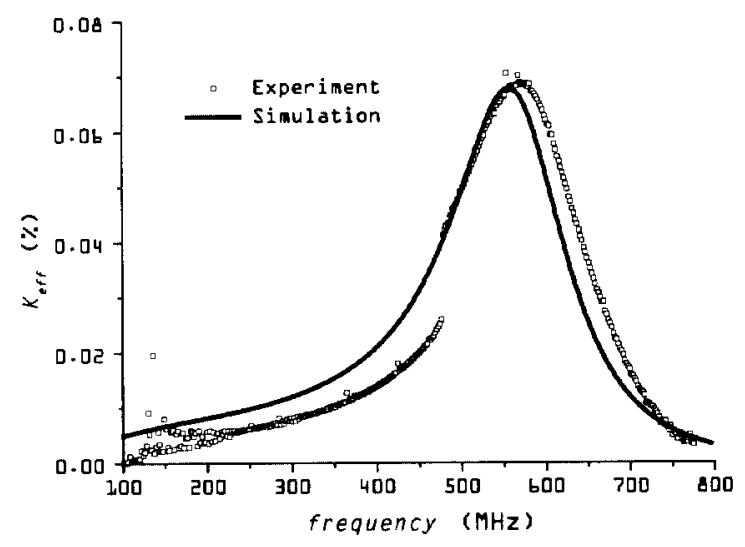

Fig 5. SPRF distribution on $\mathrm{ZnO}$ /fused quartz.

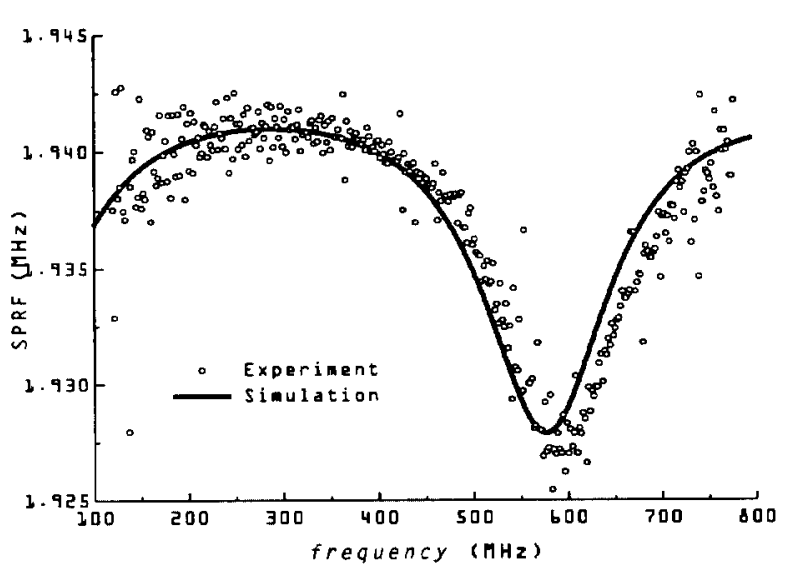

Fig 6. $k_{e f f}^{2}(m)$ distribution of $\mathrm{ZnO} /$ fused quartz.

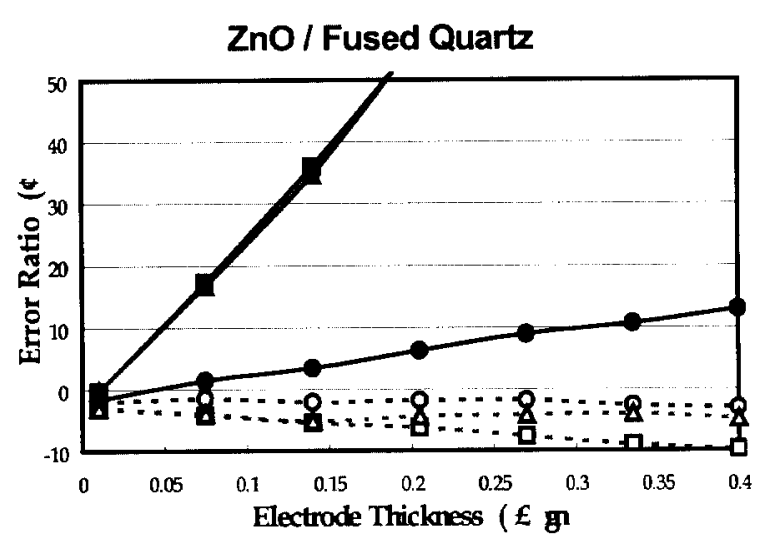

Fig 7.

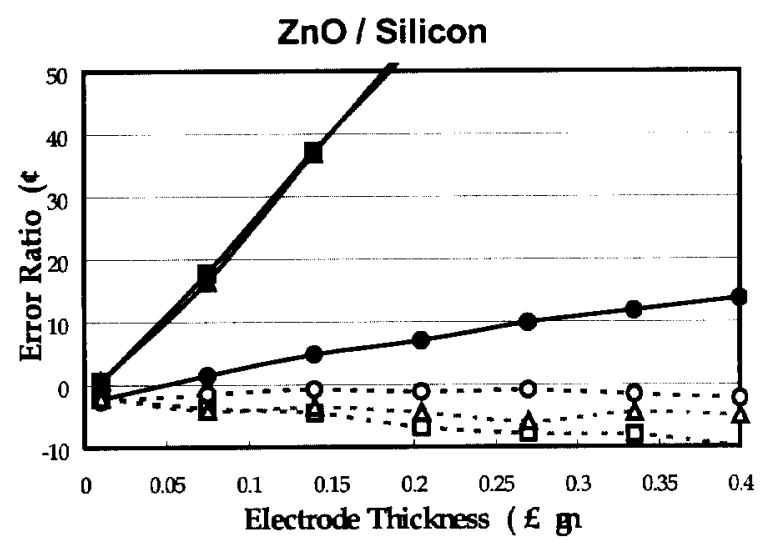

Fig 8.

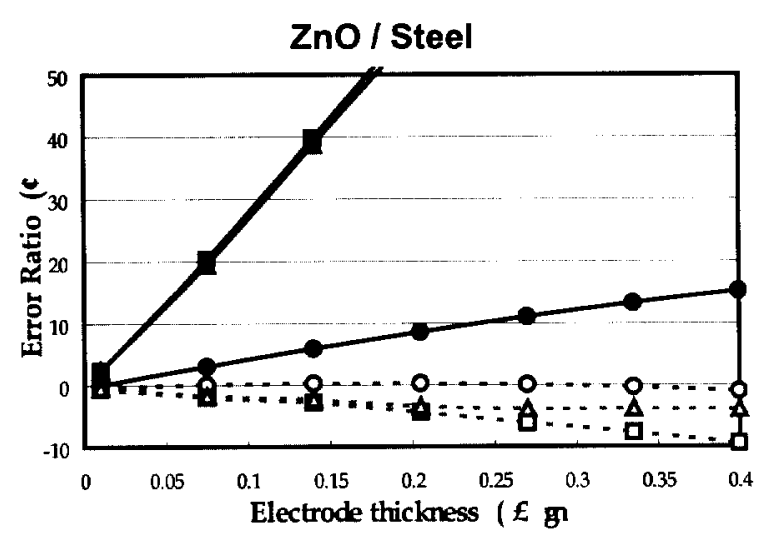

Fig 9. 


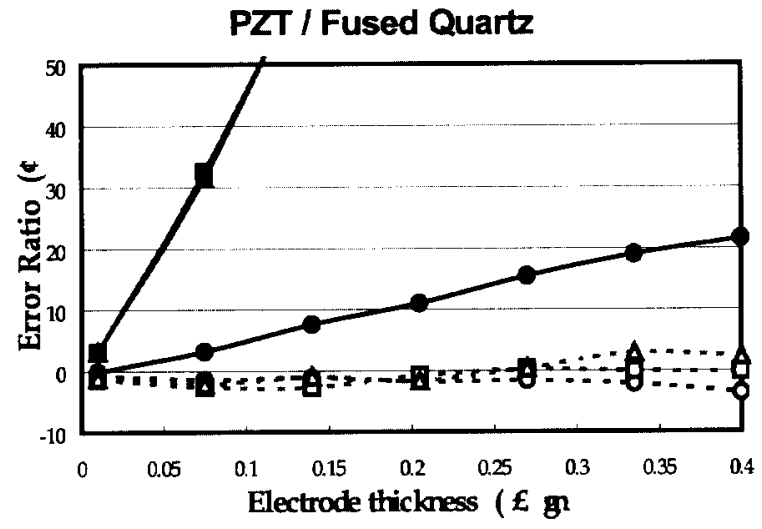

Fig 10.

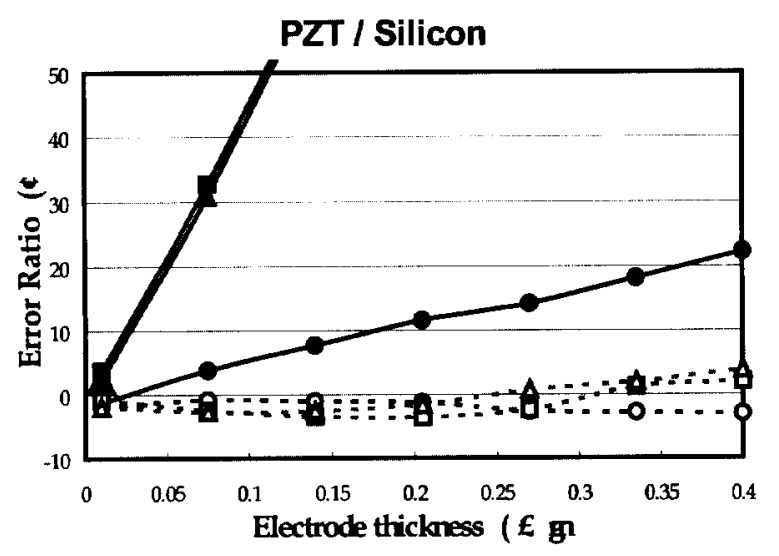

Fig 11.

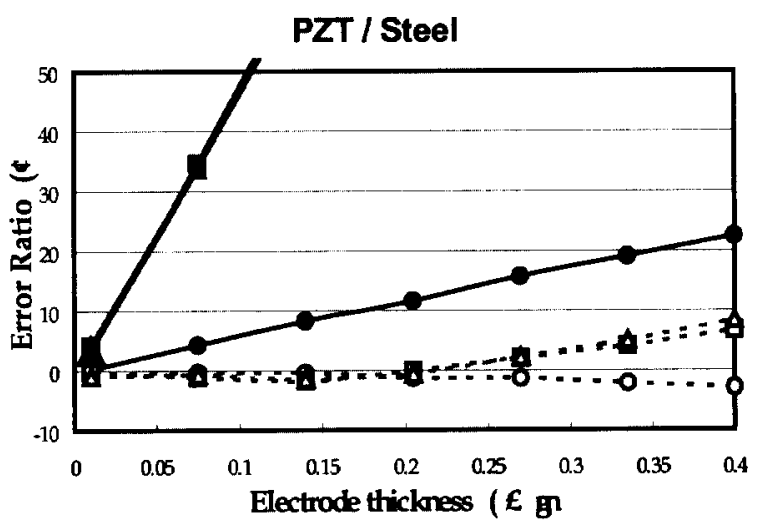

Fig 12.
Fig 7-12. Comparison of the errors caused by electrode effects when using the non-corrected formula (bold-lines) and the corrected values by modified formula (fine lines). For the six different combinations, each has three different kinds of electrodes.

*the legend of fig7 figl 2 is:

$\longrightarrow$ Al. un-modified formula
$\cdots \bigcirc-\cdots$ Al. modified formula
$-\square-$ Au un-modified formula
$\cdots \square$ Au modified formula
$\longrightarrow-W$ un-modified formula
$\cdots \Delta \cdots W$ modified formula

